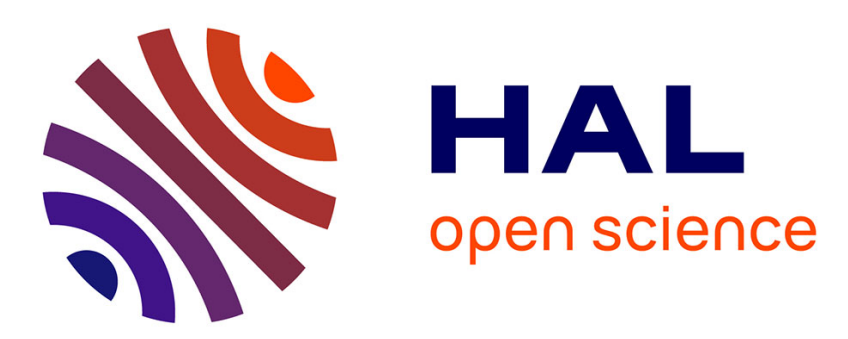

\title{
Some results on robust output feedback stabilization of nonlinear systems
}

Lorenzo Marconi, Laurent Praly

\section{To cite this version:}

Lorenzo Marconi, Laurent Praly. Some results on robust output feedback stabilization of nonlinear systems. 7th IFAC Symposium on Nonlinear Control Systems, Aug 2007, Pretoria, South Africa. pp.551-556. hal-00576958

\section{HAL Id: hal-00576958 \\ https: / hal-mines-paristech.archives-ouvertes.fr/hal-00576958}

Submitted on 15 Mar 2011

HAL is a multi-disciplinary open access archive for the deposit and dissemination of scientific research documents, whether they are published or not. The documents may come from teaching and research institutions in France or abroad, or from public or private research centers.
L'archive ouverte pluridisciplinaire HAL, est destinée au dépôt et à la diffusion de documents scientifiques de niveau recherche, publiés ou non, émanant des établissements d'enseignement et de recherche français ou étrangers, des laboratoires publics ou privés. 


\title{
SOME RESULTS ON ROBUST OUTPUT FEEDBACK STABILIZATION OF NONLINEAR SYSTEMS 1
}

\author{
Lorenzo Marconi* Laurent Praly** \\ * CASY-DEIS Università di Bologna - Italy \\ ** École des Mines de Paris, Fontainebleau - France.
}

\begin{abstract}
This paper aims at extending the results presented in (Teel and Praly, 1995) about the design of output feedback stabilizers for systems in normal form starting from Uniformly Completely Observable (UCO) state feedback control laws in two main directions: first, we show how output feedback asymptotic stabilization can be achieved even without requiring local exponential stability of the state feedback UCO-based loop and without designing an explicit local nonlinear observer. Second, we show how to design the output feedback stabilizer starting from an UCO state feedback control law which is not vanishing on the desired asymptotic attractor which, as a consequence, may be not invariant for the original controlled system. Key tools in achieving this goal are the ones developed in (Marconi et al., 2006) in a context strongly inspired by output regulation problems. Copyright (c) 2007 IFAC
\end{abstract}

Keywords: Output feedback stabilization, robust control, observers, internal model.

\section{INTRODUCTION}

The problem of output feedback stabilization in the large for nonlinear systems has been the subject of a remarkable research attempt in the last twenty years or so (see (Isidori, 1999)). The attempt has been initially turned to identify systematic design procedures for state-feedback stabilization of specific classes of nonlinear systems. To this respect it is worth mentioning the research current focused on back-stepping design procedures for lower triangular nonlinear systems with (Kanellakopoulos et al., 1992) for the global case and (Teel and Praly, 1995) for the semiglobal case. Then, the attention of the researchers shifted to the identification of partial-state and output

1 This work was supported by MIUR. Corresponding author: Lorenzo Marconi. Tel. +39 051 2093788, Fax. +39 051 2093073, email: lmarconi@deis.unibo.it. feedback stabilization algorithms mainly addressed in a semi-global sense due to intrinsic limitations characterizing this class of problems (see (Mazenc et al., 1994)). Within the number of research directions undertaken in this field, a special role has been played by nonlinear separation principles based on the design of an explicit full state observer (see (Teel and Praly, 1994)). The main limitation of this approach is, thought, the lack of a guaranteed level of robustness of the resulting controller mainly due to the absence of a wellestablished theory of robust nonlinear state observers. Furthermore, full state observability of the controlled plant is not, in principle, a necessary condition for output feedback stabilization. A step forward to overcome these limitations has been taken in (Teel and Praly, 1995) with the definition of Uniform Completely Observable (UCO) state-feedback control law, namely a stabilizing state dependent law which can be expressed as 
nonlinear function of the control input and output and their time derivatives. In this case the issue is not to estimate the full-state but rather to reproduce directly the stabilizing law through the estimation of the input-output derivatives. This, in (Teel and Praly, 1995), has been achieved by a mix of back-stepping and partial-state observation techniques yielding an output feedback stabilizer which is robust in the measure in which the UCO function does not depend on the uncertainties and the UCO control law is vanishing on the desired asymptotic attractor. Furthermore the asymptotic features of the resulting closed-loop system are subjected to the requirement that the initial statefeedback UCO-based closed-loop system is locally exponential stable. Practical stability must be accepted otherwise (see also (Byrnes et al., 2005) at this regard). The latter limitation may be overtaken with the design of a local nonlinear observer in the spirit of (Teel and Praly, 1994) by resuming again nonlinear separation principles. However, so-doing, the same limitations outlined before come out.

This work frames in the previous UCO context and aims at removing the main limitations illustrated before. More specifically, by means of the mathematical tools which have been developed in (Marconi et al., 2006), we show how the design of a dynamic output feedback control law which asymptotically stabilizes a compact attractor can be obtained by starting from a UCO statefeedback control law which does not necessarily stabilize in exponential way the desired asymptotic attractor and which is not necessarily vanishing on it. We will show that these limitations can be removed by means of design techniques, developed in the context of output regulation theory, aiming to robustly get rid of interconnections terms between nonlinear dynamics arising in the stability analysis which are not vanishing on the desired asymptotic attractor and which, as a consequence, can not be dominated only by means of high-gain. This will lead to identify a dynamic back-stepping and an extended partialstate observer algorithms which embed solution techniques typical of internal model-based design.

\section{BASIC MATHEMATICAL TOOLS}

In this section we review the basic tools proposed in (Marconi et al., 2006) and we present some new ones instrumental for the scope of this paper.

Consider a smooth nonlinear system described by

$$
\begin{aligned}
\dot{w} & =s(w) & & w \in W \subset \mathbb{R}^{s} \\
\dot{z} & =f(w, z) & & z \in \mathbb{R}^{n}
\end{aligned}
$$

in which $W$ is a compact set assumed to be invariant for $\dot{w}=s(w)$. As a particular case, the signals $w(t)$ generated by $\dot{w}=s(w)$ may be constant signals, i.e. $s(w) \equiv 0$, namely constant parameters taking value in the set $W$ and affecting the system $\dot{z}=f(w, z)$. The first crucial tool is presented in the next proposition (see Proposition 1 in (Marconi et al., 2006)).

Proposition 2.1. Let $\mathcal{B} \subset \mathbb{R}^{s} \times \mathbb{R}^{n}$ be locally asymptotically stable for the system (1) with domain of attraction $M \times \mathcal{D}, \mathcal{D} \subset \mathbb{R}^{n}$. Let $q: W \times$ $\mathbb{R}^{n} \mapsto \mathbb{R}$ be a smooth real-valued function and consider the system

$$
\dot{x}=F x+G q(w, z) \quad x \in \mathbb{R}^{m}
$$

with $(F, G) \in \mathbb{R}^{m \times m} \times \mathbb{R}^{m \times 1}$ a controllable pair and $F$ Hurwitz. Then there exists at least one continuous map $\tau: \mathbb{R}^{s+n} \rightarrow \mathbb{R}^{m}$ solution of the PDE

$$
L_{(1)} \tau(w, z)=F \tau(w, z)+G q(w, z)
$$

where $L_{(1)} \tau(w, z)$ is the Lie derivative at $(w, z)$ of $\tau$ along (1) and the set

$$
\begin{array}{r}
\operatorname{graph}\left(\left.\tau\right|_{\mathcal{B}}\right)=\left\{(w, z, x) \in \mathcal{B} \times \mathbb{R}^{m}:\right. \\
x=\tau(w, z)\}
\end{array}
$$

is locally asymptotically stable for $(1),(2)$ with domain of attraction $M \times \mathcal{D} \times \mathbb{R}^{m}$. Furthermore the set in question is locally exponentially stable for (1), (2) if $\mathcal{B}$ is such for (1).

The second tool is presented in the next lemma (see Propositions 2 and 3 of (Marconi et al., 2006)) strictly related to the statement of the previous proposition.

Lemma 2.1. Consider system (1), (2) under the conditions expressed in Proposition 2.1. Let $m \geq$ $2(s+n)+2$. There exists an $\ell>0$ and a set $\mathcal{S} \subset \mathbb{C}$ of zero Lebesgue measure such that if ${ }^{2}$ $\sigma(F) \in \mathbb{C}_{\ell}^{-} \backslash \mathcal{S}$ then for any compact set $\mathcal{R} \subset W \times$ $\mathbb{R}^{n}$ there exists a continuous function $\gamma: \mathbb{R}^{m} \rightarrow \mathbb{R}$ such that

$$
\gamma \circ \tau(w, z)+q(w, z)=0 \quad \forall(w, z) \in \mathcal{R} .
$$

Following the proof of Proposition 3 in (Marconi et al., 2006), it turns out the requirement of choosing $F$, besides Hurwitz, with a certain stability margin fixed in the previous proposition by the positive real number $\ell$, represents only a technical assumption needed to guarantee that the function $\tau$ is $C^{1}$. In this sense the assumption in question must be not confused with a "high gain" requirement on the choice of $F$. In other words any choice

\footnotetext{
${ }^{2} \sigma(F)$ denotes the spectrum of $F$ while $\mathbb{C}_{\ell}:=\{\lambda \in \mathbb{C}$ : $\operatorname{Re} \lambda<-\ell\}$.
} 
of $F$ such that the associated $\tau$ is differentiable is an appropriate choice. Furthermore note how the previous lemma only claims the existence of a continuous $\gamma$ fulfilling (4). As, in the course of the paper, we shall need to strengthen the regularity of the $\gamma$ satisfying (4), we introduce the following definition.

Definition. In the framework of Proposition 2.1 and Lemma 2.1, the triplet $((1), q(\cdot), \mathcal{B})$ is said to be regular if there exist a suitable choice of a pair $(F, G)$ and locally Lipschitz functions $\gamma$ and $\tau$ satisfying the PDE (3) and (4) for some $\mathcal{R}$ such that $\mathcal{B} \subset$ int $\mathcal{R}$. The triplet is said to be strongly regular if $\gamma$ and $\tau$ are smooth. $\triangleleft$

Remark. Even though the formulation of precise conditions under which the function $\gamma$ is more than continuous is beyond the scope of this paper, it turns out that a sufficient condition for $\gamma$ to be regular is that system (1) with output $q(w, z)$ is observable in a proper sense. Details in this direction will be discussed in future works on this subject.

To the previous results we add a new tool, which will play a crucial role in the following, formalized in the next proposition.

Proposition 2.2. Let $\mathcal{B} \subset \mathbb{R}^{s} \times \mathbb{R}^{n}$ be locally asymptotically stable for the system (1) with domain of attraction $W \times \mathcal{D}, \mathcal{D} \subset \mathbb{R}^{n}$. For any compact set $\mathcal{R} \subset \mathbb{R}^{s+n}$ such that $\mathcal{B} \subset$ int $\mathcal{R}$, there exists a compact set $\mathcal{B}_{e}$ satisfying $\mathcal{B} \subseteq \mathcal{B}_{e} \subset \mathcal{R}$ which is locally exponentially stable for (1) with the same domain of attraction $W \times \mathcal{D}$.

Note that, the joint application of Propositions 2.1 and 2.2 and Lemma 2.1 yield that if $\mathcal{B}$ is a locally asymptotically stable set for (1) with domain of attraction $W \times D$, then there exists a compact set $\mathcal{B}_{e} \supseteq \mathcal{B}$ and continuous functions $\gamma$ and $\tau$ such that $\operatorname{graph}\left(\left.\tau\right|_{\mathcal{B}_{e}}\right)$ is locally exponentially stable for (1),(2) and $\gamma \circ \tau(w, z)+q(w, z)=0$ for all $(w, z) \in \operatorname{graph}\left(\left.\tau\right|_{\mathcal{B}_{e}}\right)$.

\section{ROBUST ASYMPTOTIC STABILIZATION OF COMPACT SETS}

In this paragraph we show how the tools presented in Section 2 lend themselves to be useful to solve challenging robust output feedback stabilization problems. The setting considered here is the one proposed in (Teel and Praly, 1995) in which output feedback stabilizers are designed starting from UCO state feedback control laws.

Consider the system

$$
\begin{aligned}
\dot{w} & =s(w) & & w \in \mathbb{R}^{s} \\
\dot{z} & =A(w, z, u) & & z \in \mathbb{R}^{n}, u \in \mathbb{R} \\
y & =C(w, z) & & y \in \mathbb{R}
\end{aligned}
$$

with initial conditions $w(0) \in W, z(0) \in Z$, $W \subset \mathbb{R}^{s}, Z \subset \mathbb{R}^{n}$ compact sets. As above, we assume $W$ is an invariant set for the first dynamics in (5). We recall (see (Teel and Praly, 1995)) that a function $\bar{u}$ is said to be UCO with respect to (5) if there exist two integers $n_{y}, n_{u}$ and a $\mathcal{C}^{1}$ function $\Psi$ such that, for each solution of

$$
\begin{aligned}
\dot{w} & =s(w) \\
\dot{z} & =A\left(w, z, u_{0}\right) \\
\dot{u}_{i} & =u_{i+1} \quad 0=1, \ldots, n_{u}-1 \\
\dot{u}_{n_{u}} & =v
\end{aligned}
$$

we have, for all $t$ where the solution makes sense,

$\bar{u}(w(t), z(t))=\Psi\left(y(t), y^{(1)}(t), \ldots, y^{\left(n_{y}\right)}(t)\right.$,

where $y^{(i)}(t)$ denotes the $i$ th derivative of $y$ at time $t$. In this framework the following theorem, which extends in several meaningful directions Theorem 1.1 of (Teel and Praly, 1995), can be proved.

Theorem 3.1. Consider system (5) and assume the existence of a compact set $\mathcal{A} \in \mathbb{R}^{s+n}$ and of a function $\bar{u}(w, z)$ such that

(a) $\mathcal{A}$ is locally asymptotically stable for system (5) with $u=\bar{u}(w, z)$ with domain of attraction $W \times \mathcal{D}, Z \subset \mathcal{D}$;

(b) $\bar{u}(w, z)$ is $\mathrm{UCO}$ with respect to (5).

Then there exist ${ }^{3} v>0$, compact sets $C, \mathcal{C} \subset \mathbb{R}^{v}$ and an output feedback controller of the form

$$
\dot{\zeta}=\Phi(\zeta, y) \quad u=P(\zeta, y), \quad \zeta \in \mathbb{R}^{v}
$$

such that $\mathcal{A} \times \mathcal{C}$ is locally asymptotically stable for the closed-loop system (5)-(8) with a domain of attraction $W \times \mathcal{D}^{\prime}$ where $Z \times C \subset \mathcal{D}^{\prime}$.

This theorem extends the result presented in Theorem 1.1 of (Teel and Praly, 1995) in three directions. First, note that we are dealing with stabilization of compact attractors for systems evolving on closed sets (as system (5) is as a consequence of the fact that $W$ is invariant for $\dot{w}=s(w))$. This is a technical improvement on which, though, we would not like to put the emphasis. Second, note that the UCO control law $\bar{u}(w, z)$ is not required to be vanishing on the attractor $\mathcal{A}$ which, as a consequence, is not required to be forward invariant for the open loop system (5) with $u \equiv$

\footnotetext{
3 modulo a few technical assumptions precisely specified later.
} 
0. Finally, the previous theorem claims that by means of a pure output feedback controller we are able to restore the asymptotic properties of an UCO controller without relying upon exponential stability requirements of the latter and robustly with respect to uncertain parameters. The last two extensions are conceptually very much relevant and their validity strongly relies upon the tools presented in Section 2. Following the main laying of (Teel and Praly, 1995), the proof of the claim is divided in two subsections which contain results interesting on their own.

\subsection{Robust asymptotic back-stepping}

In this part we discuss how the UCO control law $\bar{u}$ can be robustly back-step through the chain of integrators of (6). The results in this direction are meaningful extensions of the analogous results of (Teel and Praly, 1995) in the measure in which one considers the fact that $\bar{u}(w, z)$ is not vanishing on the attractor and that $\mathcal{A}$ is not necessarily locally exponential stable for the closed loop system.

Let $\ell_{u} \geq n_{u}$ be such that, considering the extended system

$$
\begin{aligned}
\dot{w} & =s(w) & & \dot{z}=A\left(w, z, u_{0}\right) \\
\dot{u}_{i} & =u_{i+1} & & i=0, \ldots, \ell_{u}-1 \\
\dot{u}_{\ell_{u}} & =v & &
\end{aligned}
$$

there exist functions $C_{i}$ such that

$$
y^{(i)}=C_{i}\left(w, z, u_{0}, \ldots, u_{\ell_{u}}\right) \quad i=0, \ldots, n_{y}+1 .
$$

We show that the existence of the static UCO stabilizer for (5) implies the existence of a dynamic stabilizer for (9) using the partial state $u_{i}, i=0, \ldots, \ell_{u}$, and the output derivatives $y^{(i)}$, $i=1, \ldots, n_{y}$. This is formally proved in the next theorem in which we need referring to the system

$$
\dot{w}=s(w) \quad \dot{z}=A(w, z, \bar{u}(w, z))
$$

and to the function

$q_{0}(w, z):=-\frac{\partial \bar{u}}{\partial w} s(w)-\frac{\partial \bar{u}}{\partial z} A(w, z, \bar{u}(w, z))$.

Theorem 3.2. Assume that the triplet $\left((10), q_{0}(\cdot)\right.$, $\mathcal{A})$ is strongly regular. Then there exist $v>0$, compact sets $X, \mathcal{X} \subset \mathbb{R}^{\ell_{u}+v+1}$ and a controller of the form

$$
\begin{aligned}
& \dot{\chi}=\varphi\left(\chi, y, y^{(1)}, \ldots, y^{\left(n_{y}\right)}, u_{0}, \ldots, u_{n_{u}}\right) \\
& v=\varrho\left(\chi, y, y^{(1)}, \ldots, y^{\left(n_{y}\right)}, u_{0}, \ldots, u_{n_{u}}\right)
\end{aligned}
$$

$\chi \in \mathbb{R}^{v}$, such that system $\mathcal{A} \times \mathcal{X}$ is locally asymptotically stable for the closed loop system (9), (11) with domain of attraction $W \times \mathcal{D}^{\prime}$ with $Z \times X \subset \mathcal{D}^{\prime}$.

Proof. By employing the strong regularity of the triplet $\left((10), q_{0}(w, z), \mathcal{A}\right)$, let $(F, G) \in \mathbb{R}^{v \times v} \times$
$\mathbb{R}^{v \times 1}, v>0$, be a controllable pair and $\gamma$ a smooth function such that (see Lemma 2.1)

$$
\gamma \circ \tau(w, z)+q_{0}(w, z)=0 \quad \forall(w, z) \in \mathcal{R}
$$

in which $\tau$ satisfies the PDE

$$
L_{(10)} \tau(w, z)=F \tau(w, z)+G q_{0}(w, z)
$$

and $\mathcal{R}$ is a compact set such that $\mathcal{A} \subset \operatorname{int} \mathcal{R}$. As candidate controller choose a system of the form

$$
\begin{aligned}
\dot{\eta}= & F \eta+G u_{1} \quad \eta \in \mathbb{R}^{v} \\
v= & \kappa^{\ell_{u}+1}\left(a_{0}\left(u_{0}-\bar{u}\right)+a_{1} \frac{u_{1}-\gamma(\eta)}{\kappa}+\ldots\right. \\
& \left.+a_{\ell_{u}} \frac{u_{\ell_{u}}-\gamma^{\left(\ell_{u}-1\right)}(\eta)}{\kappa^{\ell_{u}}}\right)+\gamma^{\left(\ell_{u}\right)}(\eta) \\
\bar{u}= & \Psi\left(y, y^{(1)}, \ldots, y^{\left(n_{y}\right)}, u_{0}, \ldots, u_{n_{u}}\right)
\end{aligned}
$$

in which $a_{i}, i=1, \ldots, \ell_{u}$, are arbitrary coefficients such that the roots of

$$
\lambda^{\ell_{u}+1}+a_{\ell_{u}} \lambda^{n_{u}}+\ldots+\lambda a_{1}+a_{0}=0
$$

have negative real part, $\kappa$ is a design parameter and $\gamma^{(i)}(\eta)$ denotes the $i$-th time derivative of the function $\gamma(\eta), i=1, \ldots, \ell_{u}$. Note that there exist smooth functions $\gamma_{i}$ such that $\gamma^{(i)}(\eta)=$ $\gamma_{i}\left(\eta, u_{1}, \ldots, u_{i}\right), i=1, \ldots, \ell_{u}$. By changing coordinates as

$$
\begin{aligned}
u_{0} & \mapsto \xi_{0}=u_{0}-\bar{u}(w, z) \\
u_{i} & \mapsto \xi_{i}=\frac{u_{i}-\gamma_{i-1}\left(\eta, u_{1}, \ldots, u_{i-1}\right)}{\kappa^{i}} \\
\eta & \mapsto x=\eta-G \xi_{0}
\end{aligned}
$$

with $i=1, \ldots, \ell_{u}$, it turns out that system (9), (13) with the identity (7), transforms as

$$
\begin{aligned}
\dot{w} & =s(w) \\
\dot{z} & =A(w, z, \bar{u}(w, z))+N_{1}\left(w, z, \xi_{0}\right) \\
\dot{x} & =F x-G q_{0}(w, z)+N_{2}\left(w, z, \xi_{0}\right) \\
\dot{\xi} & =\kappa H \xi+D B(w, z, x)+D N_{3}\left(w, z, x, \xi_{0}\right)
\end{aligned}
$$

in which $\xi=\left(\xi_{0}, \ldots, \xi_{\ell_{u}}\right), H$ is an Hurwitz matrix, $D=\left(\begin{array}{lll}1 & 0 \ldots 0\end{array}\right)^{\mathrm{T}}, N_{1}(\cdot), N_{2}(\cdot)$ and $N_{3}(\cdot)$ are locally Lipschitz functions vanishing at $\xi_{0}=0$ for all $(w, z, x) \in \mathbb{R}^{s} \times \mathbb{R}^{n} \times \mathbb{R}^{v}, B(w, z, x)=\gamma(x)+$ $q_{0}(w, z)$. Consider now the triangular system

$$
\begin{aligned}
\dot{w} & =s(w) \quad \dot{z}=A(w, z, \bar{u}(w, z)) \\
\dot{x} & =F x-G q_{0}(w, z)
\end{aligned}
$$

By assumption the set $\mathcal{A}$ is locally asymptotically stable for the first two dynamics of (15) with domain of attraction $W \times \mathcal{D}$. By Proposition 2.2 there exists a set $\mathcal{A}_{e} \subseteq \mathcal{R}$ (with $\mathcal{R}$ the compact set characterizing (12)) which is locally exponentially stable for the first two dynamics of (15) with the same domain of attraction $W \times$ 
$\mathcal{D}$. By this Proposition 2.1 guarantees that the set $\operatorname{graph}\left(\left.\tau\right|_{\mathcal{A}_{e}}\right)$ is locally exponentially stable for (15) with domain of attraction $W \times \mathcal{D} \times \mathbb{R}^{v}$. Furthermore by (12) it turns out that

$$
B(w, z, x)=0 \quad \forall(w, z, x) \in \operatorname{graph}\left(\left.\tau\right|_{\mathcal{A}_{e}}\right) .
$$

Hence system (14) fits in the framework of Lemma A.1 by which it is possible to conclude that for any compact set $X \subset \mathbb{R}^{v}$ and $\Xi \subset \mathbb{R}^{\ell_{u}}$ there exists a $\kappa^{\star}>0$ such that for all $\kappa \geq \kappa^{\star}$ the set $\operatorname{graph}\left(\left.\tau\right|_{\mathcal{A}_{e}}\right) \times\{0\}$ is locally exponentially stable for (14) with domain of attraction $W \times \mathcal{D}^{\prime}$ with $Z \times X \times \Xi \subset \mathcal{D}^{\prime}$. From this, by reasonings based on (Sontag, 2003) (omitted for reasons of space), the claim of the theorem follows. $\triangleleft$

Remark. It is interesting to note that the role of the $\eta$-dynamics in (13), and specifically of its output $\gamma(\cdot)$, is to compensate for the term $q_{0}(w, z)$, not vanishing on the compact attractor $\mathcal{A} \subset \mathcal{A}_{e}$, which represents a "junk" between the $(w, z)$ and the $\xi$ dynamics in (14). Here is where the tools proposed in Section 2 play a role. In this sense the relevance of the previous result is to provide a (robust) dynamic back-stepping procedure able to get rid of interconnection terms which are not necessarily vanishing on the desired asymptotic attractor and, as a consequence, which can not even be dominated with high-gain.

\subsection{The extended Dirty Derivatives Observer}

In this part we show how the knowledge of the output derivatives in the UCO law can be substituted by suitable estimates. As above, the relevance and novelty of the result with respect to (Teel and Praly, 1995) rely in the fact the UCO control law is not necessarily vanishing on the attractor and it is not expected to locally exponentially stabilize $\mathcal{A}$.

In particular, motivated by the result presented in the previous section, given a compact set $\mathcal{A} \subset$ $\mathbb{R}^{s+n}$, we assume the existence of positive constants $v, n_{y}, \ell_{u}$, with $n_{y} \leq \ell_{u}$, of compact sets $X$, $\mathcal{X} \subset \mathbb{R}^{v+\ell_{u}+1}$ and of a controller of the form

$$
\begin{aligned}
\dot{\chi} & =\varphi\left(\chi, y, y^{(1)}, \ldots, y^{\left(n_{y}\right)}, u_{0}, \ldots, u_{n_{u}}\right) \\
\dot{u}_{i} & =u_{i+1} \quad i=0, \ldots, \ell_{u}-1 \\
\dot{u}_{\ell_{u}} & =\varrho\left(\chi, y, y^{(1)}, \ldots, y^{\left(n_{y}\right)}, u_{0}, \ldots, u_{n_{u}}\right) \\
u & =u_{0}
\end{aligned}
$$

such that $\mathcal{A} \times \mathcal{X}$ is locally asymptotically stable for the closed loop system (5)-(16) with a domain of attraction $W \times \mathcal{D}$ where $Z \times X \subset \mathcal{D}$. As above note that there exist smooth functions $C_{i}(\cdot)$ such that, along the solutions of the closed loop system $(5),(16), y^{(i)}(t)=C_{i}\left(w, z(t), u_{0}(t), \ldots, u_{\ell_{u}}(t)\right)$ for all $i=0, \ldots, n_{y}+1$ and for all $t$ where the solution makes sense.

Theorem 3.3. Assume that the triplet ((5) (16), $\left.C_{n_{y}+1}(\cdot), \mathcal{A} \times \mathcal{X}\right)$ is regular. There exist $\varpi \geq$ $v+\ell_{u}$, compact sets $C, \mathcal{C} \subset \mathbb{R}^{\varpi}$ and an output feedback controller of the form

$$
\dot{\zeta}=\Phi(\zeta, y) \quad u=P(\zeta, y), \quad \zeta \in \mathbb{R}^{\varpi}
$$

such that $\mathcal{A} \times \mathcal{C}$ is locally asymptotically stable for the closed loop system (5)-(17) with a domain of attraction $W \times \mathcal{D}^{\prime}$ where $Z \times C \subset \mathcal{D}^{\prime}$.

Proof. Consider the extended ${ }^{4}$ dirty derivatives observer

$\dot{\hat{y}}_{i}=\hat{y}_{i+1}+L \ell_{i}\left(y-\hat{y}_{0}\right) \quad i=0, \ldots, n_{y}-1$

$\dot{\hat{y}}_{n_{y}}=L^{n_{y}+1} \ell_{n_{y}}\left(y-\hat{y}_{0}\right)-\gamma(\eta)$

$\dot{\eta}=F \eta+G\left[\gamma(\eta)-L^{n_{y}+1} \ell_{n_{y}}\left(y-\hat{y}_{0}\right)\right]$

with $\eta \in \mathbb{R}^{m}$, where $L>1$ is a design parameter, $\ell_{i}, i=0, \ldots, n_{y}$, are such that the roots of

$$
\lambda^{n_{y}+1}+\ell_{n_{y}} \lambda^{n_{y}}+\ldots+\lambda \ell_{1}+\ell_{0}=0
$$

have negative real part, and $m,(F, G)$ and $\gamma(\cdot)$ have to be chosen. Consider now the output feedback controller

$$
\begin{aligned}
\dot{\chi} & =\varphi_{s}\left(\chi, y, \hat{y}_{1}, \ldots, \hat{y}_{n_{y}}, u_{0}, \ldots, u_{\ell_{u}}\right) \\
\dot{u}_{i} & =u_{i+1} \quad i=0, \ldots, \ell_{u}-1 \\
\dot{u}_{\ell_{u}} & =\varrho_{s}\left(\chi, y, \hat{y}_{1}, \ldots, \hat{y}_{n_{y}}, u_{0}, \ldots, u_{\ell_{u}}\right) \\
u & =u_{0}
\end{aligned}
$$

in which $\varphi_{\mathrm{s}}(p)=\varphi(p)$ if $|\varphi(p)| \leq \mathrm{s}$ and $\varphi_{\mathrm{s}}(p)=\mathrm{s}$. $\operatorname{sgn}(\varphi(p))$ otherwise, and $\varrho_{\mathrm{s}}(p)=\varrho(p)$ if $|\varrho(p)| \leq \mathrm{s}$ and $\varrho_{\mathrm{s}}(p)=\mathrm{s} \cdot \operatorname{sgn}(\varrho(p))$ otherwise.

Let $y_{d}=\operatorname{col}\left(y, y^{(1)}, \ldots, y^{\left(n_{y}\right)}\right), \hat{y}=\operatorname{col}\left(\hat{y}_{0}, \hat{y}_{1}\right.$, $\left.\ldots, \hat{y}_{n_{y}}\right)$ and consider the change of variables

$$
\hat{y} \mapsto e=D_{L}\left(y_{d}-\hat{y}\right)
$$

$D_{L}=\operatorname{diag}\left(L^{n_{y}}, L^{n_{y}-1}, \ldots, 1\right)$ and $\eta \mapsto x=\eta-$ $G e_{n_{y}}$. In this coordinate setting, by denoting

$$
\mathbf{z}=\operatorname{col}\left(z u_{0} \ldots u_{\ell_{u}} \xi\right),
$$

the overall closed-loop system reads in compact form as

$$
\begin{aligned}
\dot{w} & =s(w) \\
\dot{\mathbf{z}} & =f(w, \mathbf{z})+\Delta(w, \mathbf{z}, e) \\
\dot{x} & =F x-G C_{n_{y}+1}(w, \mathbf{z})+F G e_{n_{y}} \\
\dot{e} & =L H e+B Q_{1}(w, \mathbf{z}, x)+B Q_{2}(x, e)
\end{aligned}
$$

in which $H$ is Hurwitz, $B=\left(\begin{array}{llll}0 & \ldots & 0 & 1\end{array}\right)^{\mathrm{T}}$, $Q_{1}(w, \mathbf{z}, x)=C_{n_{y}+1}(w, \mathbf{z})+\gamma(x), Q_{2}(x, e)=$

\footnotetext{
4 The adjective extended is to emphasize the presence of the additional dynamic $\dot{\eta}$ besides the "conventional" estimate dynamics of output derivatives.
} 
$\gamma\left(x+G e_{n_{y}}\right)-\gamma(x), \operatorname{col}(s(w), f(w, z))$ is a compact representation of the vector fields in (5), (16), $\Delta(w, \mathbf{z}, e)=\operatorname{col}\left(0, \Delta_{1}(\cdot), \Delta_{2}(\cdot)\right)$ with $\Delta_{2}(\cdot)=$ $\varphi_{\mathrm{s}}(\cdot)-\varphi(\cdot)$ and $\Delta_{1}(\cdot)=\varrho_{\mathrm{s}}(\cdot)-\varrho(\cdot)$ and where, with a mild abuse of notation, we have denoted $C_{n_{y}+1}(w, \mathbf{z})=C_{n_{y}+1}\left(w, z, u_{0}, \ldots, u_{\ell_{u}}\right)$. Clearly $Q_{2}(x, 0)=0$ for all $x \in \mathbb{R}^{m}$.

The remarkable feature of this system is that, by assumption, system

$$
\dot{w}=s(w) \quad \dot{\mathbf{z}}=f(w, \mathbf{z})
$$

has a locally asymptotically set $\mathcal{A} \times \mathcal{X}$ with domain of attraction $W \times \mathcal{D}$ with $Z \times X \subset \mathcal{D}$. In particular, by taking $\mathcal{R} \subset \mathbb{R}^{s+n+\ell_{u}+\varpi}$ an arbitrary compact set such that $\mathcal{A} \times \mathcal{X} \subset \operatorname{int} \mathcal{R}$ and $\mathcal{R} \subset W \times \mathcal{D}$, Proposition 2.2 guarantees the existence of a compact set $\mathcal{B}_{e}$, with $\mathcal{A} \times \mathcal{X} \subseteq$ $\mathcal{B}_{e} \subseteq \mathcal{R}$, which is locally exponentially stable for (21) with domain of attraction $W \times \mathcal{D}$. This, by Proposition 2.1, yields that for the system (21) augmented with

$$
\dot{x}=F x-G C_{n_{y}+1}(w, \mathbf{z})
$$

with $F$ Hurwitz, there exists a continuous function $\tau: \mathbb{R}^{s+n+\ell_{u}+\varpi} \rightarrow \mathbb{R}^{m}$ such that the set $\operatorname{graph}\left(\left.\tau\right|_{\mathcal{B}_{e}}\right)$ is locally exponentially stable for (21)-(22) with domain of attraction $W \times \mathcal{D} \times \mathbb{R}^{m}$. Furthermore, by choosing $m \geq 2\left(s+n+\ell_{u}+\varpi\right)+2$, by employing the regularity assumption of the triplet $\left((5)-(16), C_{n_{y}+1}(\cdot), \mathcal{A} \times \mathcal{X}\right)$ and Lemma 2.1 , and by fixing $(F, G)$ and a locally Lipschitz $\gamma$ so that

$$
Q_{1}(w, \mathbf{z}, \tau(w, \mathbf{z}))=0 \quad \forall(w, \mathbf{z}) \in \mathcal{R}
$$

it turns out that $Q_{1}(w, \mathbf{z}, x)=0 \forall(w, \mathbf{z}, x) \in$ $\operatorname{graph}\left(\left.\tau\right|_{\mathcal{B}_{e}}\right)$. The rest of the proof follows standard arguments which are only briefly recalled. In particular, the amplitude $\mathrm{s}$ of the saturated functions $\varphi_{\mathrm{s}}(\cdot)$ and $\varrho_{\mathrm{s}}(\cdot)$ hidden in the definition of $\Delta(\cdot)$ can be tuned so that $\Delta(w, \mathbf{z}, 0) \equiv 0$ for all $(w, \mathbf{z}) \in \mathcal{B}_{e}$ so that the set $\operatorname{graph}\left(\left.\tau\right|_{\mathcal{B}_{e}}\right) \times\{0\}$ is forward invariant for (20) (at this level a proper Lyapunov characterization of the local exponential stability of the set $\mathcal{B}_{e}$ for (21) is needed). Furthermore it turns out that system (20) fits in the framework of Lemma A.1 by which it is possible to claim the existence of an $L^{\star}>0 \mathrm{such}$ that for any $L \geq L^{\star}$ the set $\operatorname{graph}\left(\left.\tau\right|_{\mathcal{B}_{e}}\right) \times\{0\}$ is locally exponentially stable for (20) with a domain of attraction $W \times \mathcal{D}^{\prime}$ with $Z \times X \times N \times L^{n_{y}} E \subset \mathcal{D}^{\prime}$. From this, by reasonings based on (Sontag, 2003) (omitted for reasons of space), the claim of the theorem follows. $\triangleleft$

Remark. Again, as in the remark at the end of Section 3.1, note that the role of the $\eta$ dynamics in
(18), and specifically of its output $\gamma(\cdot)$, is to compensate for the term $C_{n_{y}+1}(w, \mathbf{z})$, interconnecting the $(w, \mathbf{z})$ and the $e$ dynamics in $(20)$, which is not vanishing on $\mathcal{B}_{e}$.

\section{Appendix A. AUXILIARY RESULTS}

We present a technical lemma, extension a similar result in (Teel and Praly, 1995), focused on highgain feedback interconnection of systems on closed sets.

Lemma A.1. Consider the locally Lipschitz system

$$
\begin{aligned}
\dot{w} & =s(w) & & w \in W \subset \mathbb{R}^{s} \\
\dot{x} & =f(w, x, y) & & x \in \mathbb{R}^{n} \\
\dot{y} & =\kappa H y+q(w, x, y) & & y \in \mathbb{R}^{m}
\end{aligned}
$$

with $H$ Hurwitz, $\kappa>0$ and $W$ a compact set invariant for $\dot{w}=s(w)$. For the system given by the first two dynamics of (A.1) with $y=0$ assume the existence of a compact set $\mathcal{B} \subset \mathbb{R}^{s} \times \mathbb{R}^{n}$ which is locally exponentially stable with domain of attraction $W \times \mathcal{D}, \mathcal{D}$ open subset of $\mathbb{R}^{n}$. Let $q(w, x, 0)=0$ for any $(w, x) \in \mathcal{B}$. Then for any compact set $X \subset \mathcal{D}$ and $Y \subset \mathbb{R}^{m}$ and any $r>0$, there exists a $\kappa^{\star}>0$ such that for any $\kappa \geq \kappa^{\star}$ the set $\mathcal{B} \times\{0\}$ is locally exponentially stable for (A.1) with domain of attraction $W \times \mathcal{D}_{e}$ with $X \times Y \subset \mathcal{D}_{e}$.

\section{REFERENCES}

Byrnes, C. I., F. Celani and A. Isidori (2005). Omegalimit sets for a class of nonlinear systems that are semiglobally practically stabilized. Int. J. of Robust and Nonl. Cont. 15, 315-333.

Isidori, A. (1999). Nonlinear Control Systems II. Springer Verlag. New York.

Kanellakopoulos, I., P.V. Kokotovic and A. S. Morse (1992). A toolkit for nonlinear feedback design. Systems \& Control Letters 18, 83-92.

Marconi, L., L. Praly and A. Isidori (2006). Output stabilization via nonlinear luenberger observers. to appear on SIAM J. Control Optim. (ArXiv version Ref. Math. OC/0601641 v1).

Mazenc, F., L. Praly and W. P. Dayawansa (1994). Global stabilization by output feedback: examples and counterexamples. Systems \& Control Letters 23(2), 119125.

Sontag, E. D. (2003). A remark on the converging-input converging-state property. IEEE Transaction on Automatic Control 48, 313-314.

Teel, A. R. and L. Praly (1994). Global stabilizability and observability imply semi-global stabilizability by output feedback. Systems \& Control Letters 22, 313325 .

Teel, A. R. and L. Praly (1995). Tools for semiglobal stabilization by partial state and output feedback. SIAM J. Control Optim. 33, 1443-1485. 